\title{
UNA ALIANZA ENTRE LOS OBJETIVOS DEL DESARROLLO SOSTENIBLE Y LA FORMACIÓN DE MAESTRAS EN CIUDADANÍA SOSTENIBLE
}

\author{
Recepción: 13/09/2020 | Revisión: 16/10/2020 | Aceptación: 20/11/2020
}

Rosa M. MEDIR HUERTA

Universitat de Girona

rosa.medir@udg.edu

\author{
Anna SERRA-SALVI \\ Universitat de Girona \\ anna.serra@udg.edu
}

\begin{abstract}
Resumen: Se presenta una experiencia de formación inicial del profesorado de educación primaria acerca de la asunción de los Objetivos del Desarrollo Sostenible (ODS) con la intención que constituya una actividad de formación en ciudadanía sostenible. Ante los diversos tipos de enfoques que se han utilizado en educación para la ciudadanía, de los que presentamos un breve resumen, nos situamos en la ciudadanía sostenible que puede derivarse de un aprendizaje geográfico. A lo largo del curso, se han trabajado los ODS más sociales con diversas actividades de trabajo geográfico en el aula y en la ciudad. Hemos tratado temas como la pobreza, la escasez de alimentos, el espacio público de la ciudad, los roles de género en la ciudad, el consumo responsable, la responsabilidad de las empresas, testimonios de la paz y el conflicto presentes en la ciudad. Al finalizar el curso, hemos evaluado hasta qué punto los conocimientos sobre cada uno de los ODS tratados incitan poco o mucho a los cambios en las formas de vida de los propios estudiantes, y por tanto, si contribuyen a unas pautas de ciudadanía más sostenible.
\end{abstract}

Palabras clave: ODS; ciudadanía sostenible; formación de maestros; educación para la ciudadanía; formación geográfica para maestros.

\section{AN ASSOCIATION BETWEENTHE SUSTAINABLE DEVELOPMENT GOALS ANDTHETRAINING OF TEACHERS IN SUSTAINABLE CITIZENSHIP}

\begin{abstract}
An initial training experience for primary education teachers is presented on the assumption of the Sustainable Development Goals (SDG) with the intention that it becomes a training activity in sustainable citizenship. Within the different types of approaches used in citizenship education, and of which we present a brief summary, we focus on a sustainable citizenship which can come from geographic learning. The most social SDGs have been worked on throughout the course, with various geographic activities both in the classroom and in the city. We have worked on different topics such as poverty, food shortages, public space in the city, gender roles in the city, responsible consumer behavior, corporate responsibility, testimony of the peace and conflict in the city. By the end of the course, we have measured to what extent the knowledge on the SDGs discussed have influenced and changed the lifestyles of the students themselves, and therefore, if they contribute to more sustainable citizenship guidelines.
\end{abstract}

Keywords: SDG; sustainable citizenship; teacher training; citizenship education; geographical training for teachers.

\section{UNA ALIANÇA ENTRE ELS OBJECTIUS DEL DESENVOLUPAMENT SOSTENIBLE I LA FORMACIÓ DE MESTRES EN CIUTADANIA SOSTENIBLE}

Resum: Es presenta una experiència de formació inicial del professorat d'educació primària sobre l'assumpció dels Objectius del Desenvolupament Sostenible (ODS) amb la intenció que constitueixi una activitat de formació en ciutadania sostenible. Davant dels diversos tipus d'enfocaments utilitzats en leducació per a la ciutadania, dels que presentem un breu resum, ens situem en la ciutadania sostenible que pot derivar-se d'un aprenentatge geogràfic. Al llarg del curs, s'han treballat els ODS més socials amb diverses activitats de treball geogràfic a laula i a la ciutat. Hem tractat temes com la pobresa, lescassetat d'aliments, lespai públic de la ciutat, els rols de gènere a la ciutat, el consum responsable, la responsabilitat de les empreses, testimonis de la pau i el conflicte presents a la ciutat. En finalitzar el curs, hem avaluat fins a quin punt els coneixements sobre cada un dels ODS tractats inciten poc o molt als canvis en les formes de vida dels propis estudiants, i per tant, si contribueixen a unes pautes de ciutadania més sostenible.

Paraules clau: ODS; ciutadania sostenible; formació de mestres; educació per a la ciutadania; formació geogràfica per a mestres. 


\section{Introducción}

Formación de maestros y maestras, educación para la ciudadanía sostenible, ¿cómo lo unimos? Aparentemente, y sin saber las concepciones que cada uno tenga acerca de los campos pedagógicos mencionados, creemos que una mayoría se decanta por su relación académica en la formación inicial y práctica en la vida profesional.

La didáctica de las ciencias sociales, presente en la formación de maestros/as, puede y debe ocuparse de ello. Ante este reto, se desarrolla la experiencia que presentamos en las líneas que siguen. Dentro de las ciencias sociales, la Geografía aporta mucho al concepto de ciudadanía sostenible y a la educación para la ciudadanía, en general.

En los escasos espacios formativos que nos proporciona el plan de estudios de Maestro/a, el profesorado de nuestra área de conocimiento intenta seleccionar aquello que considera más relevante en el ámbito de la Didáctica de las Ciencias Sociales. La experiencia aportada podemos llevarla a cabo solamente en el Grado de Primaria (no en el de Infantil), dentro de la asignatura obligatoria de tres créditos llamada «Sociedad y Sostenibilidad». La finalidad principal de dicha asignatura se centra en profundizar en el concepto de sostenibilidad, especialmente desde un punto de vista social. Los primeros años, lo hacíamos para desembocar en el concepto de Educación para la sostenibilidad. Desde la irrupción en nuestra sociedad de los Objetivos del Desarrollo Sostenible (ODS) de la Agenda 2030, hemos decidido reorientar nuestro programa docente para trabajar dichos objetivos y relacionarlos con la educación para la ciudadanía. Cabe decir que los y las estudiantes pueden seguir una mención (optativas) en la cual tienen dos/tres asignaturas que pueden calificarse dentro de la Educación para la Sostenibilidad. En cambio, el espacio docente para la educación para la ciudadanía (y ciudadanía sostenible) es mucho más reducido. Nuestro reto es saber crear una alianza coherente entre los ODS y la formación en ciudadanía sostenible.

\section{Evolucionando en los conceptos de educación para la ciudadanía}

Nos preguntamos: ¿ciudadanía global, ciudadanía sostenible o sencillamente educación para la ciudadanía? Las expresiones diferentes, con muchas coincidencias, se acumulan en la bibliografía relacionada con este tema. La expresión más genérica «educación para la ciudadanía» se ha abierto y ampliado a más y más perspectivas o miradas, relacionadas con la sostenibilidad y, en consecuencia, con el mundo global.

Para la didáctica de las ciencias sociales, la educación para la ciudadanía es vista como un enfoque de una enseñanza de las propias ciencias sociales centrada en los problemas sociales relevantes, en la cual la geografía ha jugado un papel determinante, ya que desde los inicios del siglo XX ha sido definida y reclamada para enseñar saberes socialmente útiles, para la enseñanza de la acción cívica y la participación democrática (Pagès, 2007). Por su parte, desde movimientos de renovación pedagógica de las tierras de Valencia se desarrolló y concretó una propuesta centrada en los problemas sociales y la didáctica de la geografía. En su fundamentada opinión «los contenidos propios de esta área de conocimiento (las ciencias sociales, geografía e historia) deben considerar esa cotidianidad para ser útiles y significativos en la explicación de la 
realidad social por parte de los alumnos» (Souto, 1998:268).

La relación entre la didáctica de las ciencias sociales y la sostenibilidad se hace evidente a medida que este concepto se asume y se esparce en los ámbitos académicos. La educación para la sostenibilidad irrumpe en el mundo educativo, en formulaciones transversales, a menudo difíciles de aplicar por los docentes, los cuales se ven abrumados por una gran cantidad de terminología y conceptos en su ámbito de conocimiento: didáctica de la geografía, didáctica de las ciencias sociales, educación para la sostenibilidad, educación para el desarrollo, educación para el desarrollo sostenible, educación para la ciudadanía, educación para la participación ciudadana, ciudadanía ambiental, ciudadanía global, educación para la ciudadanía global y muchas más temáticas relacionadas.

En la formación del profesorado, nuestro ámbito de actuación, hemos intentado siempre esclarecer este bosque terminológico, y dar algunas pautas claras para la acción didáctica en primaria como en secundaria. Justamente, desde el ámbito anglosajón y desde los años noventa del siglo pasado, para la formación del profesorado, se apuesta por una interrelación de la educación para la sostenibilidad con la educación ciudadana, para obtener una «ciudadanía ecológica» (Granados, 2004). La ciudadanía ecológica «se entiende bajo el punto de vista de la reconstrucción social y se centra en el triunvirato justicia, equidad y obligaciones, valores cruciales para la sostenibilidad y la democracia» (Granados, 2008:222). Creemos que este término no ha triunfado, o ha pasado desapercibido en nuestro entorno. Destacamos este hecho porque creemos que, por tanto, aquí, no se ha evolucionado desde un concepto de ciudadanía ecológica a una ciudadanía sostenible, como puede haber sucedido en otros países. En todo caso, sí se ha hablado algo más de «ciudadanía ambiental» (¿la podemos considerar un medio camino entre la ecológica y la sostenible?) de la mano de Michela Mayer, quien ha definido las contribuciones de la educación ambiental en la educación para la ciudadanía. La misma autora cambió la terminología, pasando a hablar de «ciudadanía glocal», entendiendo que la dimensión transnacional de los problemas socioambientales ha de ser compatible con la pertenencia a comunidades locales o cercanas, próximas en términos de identidad (Mayer, 2002). El uso de la nueva palabra «glocal» nos acerca a la corriente más actual de la ciudadanía global.

La ciudadanía sostenible definida por John Barry (2006), es mucho más ambiciosa, multifacética y se preocupa más de la justicia social, la equidad y la gobernanza democrática. Se establece que:

«esta ciudadanía se preocupa también de las causas estructurales de la degradación socioambiental, pero ahonda, sobre todo, en aquellos aspectos sociales y económicos del desarrollo sostenible como la alteridad, el respeto de los derechos humanos, la inclusión social, la igualdad, la calidad de vida, la democracia deliberativa, la participación y la buena gobernanza, la solidaridad, etc.» (Granados, 2008:223)

Ante este concepto de ciudadanía sostenible: ¿cómo debe prepararse el profesorado? En todo caso, somos conscientes que no podremos controlar la formación que reciben los y las estudiantes, futuros docentes, ya que nos podemos mover solamente en los pequeños espacios que nos ofrezca el plan de estudios. Por tanto, será una asignatura de 3 créditos ECTS obligatoria para la titulación de Maestro/a de Educación Primaria llamada 
«Sociedad y Sostenibilidad» la que nos proporciona el espacio para intentar formar a los pre-docentes en ciudadanía sostenible.

Más recientemente, desde UNESCO se interesan por la ciudadanía global y, en consecuencia, por la educación para la ciudadanía global (Global Citizenship Education, que en español traducen como Educación para la ciudadanía mundial), y la proponen como una perspectiva emergente en todos los sistemas educativos, en conexión con el desarrollo sostenible, la defensa de los derechos humanos universales, la igualdad de género, el respeto a la diversidad y la educación para la paz (Cercadillo, 2016). En palabras de UNESCO:

«la educación para la ciudadanía mundial aspira a ser un factor de transformación, inculcando los conocimientos, las habilidades, los valores y las actitudes que los educandos necesitan para poder contribuir a un mundo más inclusivo, justo y pacífico. La educación para la ciudadanía mundial adopta un enfoque polifacético, utilizando conceptos y metodologías que ya se aplican en otros ámbitos, entre ellos la educación para los derechos humanos, la educación para la paz, la educación para el desarrollo sostenible y la educación para el entendimiento internacional» (UNESCO, 2015:15)

Este enfoque, nos sirve también para preparar nuestra formación de maestros y maestras de Educación Primaria.

En los últimos años, diversos proyectos de investigación y colaborativos están trabajando en el desarrollo de la educación para la ciudadanía. Citamos el ya antiguo Proyecto IRES (Investigación y Renovación Escolar) de la Universidad de Sevilla, y sus ramificaciones, que siguen particularmente aportando conclusiones para la formación de profesorado, como las que se desprenden del trabajo de García-Pérez, de Alba-Fernández y Navarro-Medina (2015). En dicho trabajo se analizan los resultados en tres asignaturas de la formación inicial (dos de primaria y una de secundaria) que han sido diseñadas para incentivar la educación para la ciudadanía y la participación.

También se ha desarrollado el proyecto Erasmus Plus «Pedagogia della cittadinanza e formazione degli insegnanti: un'alleanza tra scuola e territorio» (en inglés, School Territory Environment Pedagogy, STEP) liderado por la Università degli studi Milano Biccoca que considera sus temáticas centrales: convivencia civil, desarrollo sostenible, patrimonio y alianza con el territorio. El proyecto ha actuado en la escuela de Primaria y en la formación del profesorado. Apuesta por una educación a partir de los problemas del territorio local, buscando puntos de vista y de pensamiento diferentes (tal y como ha buscado siempre la educación para la sostenibilidad) para exportarlos también a una dimensión global (Schenetti y Guerra, 2018). La relación entre los fenómenos sociales locales y globales constituye uno de los puntos de apoyo de nuestra propuesta formativa.

\section{Los Objetivos del Desarrollo Sostenible: un marco para la formación en ciudadanía sostenible}

Los Objetivos de Desarrollo Sostenible (ODS) constituyen un llamamiento universal a la acción para poner fin a la pobreza, proteger el planeta y mejorar las vidas y las perspectivas de las personas en todo el mundo. En 2015, todos los estados miembros de las Naciones Unidas aprobaron 17 Objetivos como parte de la Agenda 2030 para el Desarrollo Sostenible, en la cual se establece un 
plan para alcanzar los Objetivos en 15 años. La Agenda 2030 señala que no puede haber desarrollo sostenible sin paz, ni paz sin desarrollo. Y además tiene alcance universal y no se limita a los países más pobres, o a países en conflicto, aunque reconoce su especificidad, asumiendo la necesidad de que los ODS se adapten a las diferentes realidades regionales, nacionales y locales (Mesa, 2019).

El sistema educativo, y en particular, la Universidad, no puede ser ajena a tal desafío. Como docentes universitarias dedicadas a la formación de maestros y maestras hemos querido asumir el reto de dar a conocer y relacionar con la didáctica de la geografía dichos objetivos y hemos planificado nuestra asignatura de tres créditos alrededor de los ODS, en nuestro caso, trabajando los más cercanos a las ciencias sociales y a la geografía humana. Además, la adaptación de los ODS a diferentes escalas de análisis nos hace más atractivo y posible el tratamiento en nuestras aulas. Seguimos la idea de Mesa (2019:8) según la cual «la educación para la ciudadanía global es una apuesta para conformar una ciudadanía comprometida con la democracia y la justicia en el marco de la Agenda 2030 y de los Objetivos de Desarrollo Sostenible».

\subsection{Los Objetivos (ODS) seleccionados}

Tenemos 17 Objetivos de Desarrollo Sostenible, todos importantes; nuestra capacidad de actuación en la asignatura «Sociedad y Sostenibilidad», obligatoria en el plan de estudios de Maestro/a de Educación Primaria, es de un total de 12 sesiones de clase, lo que significa un curso de 16 horas presenciales. Nuestra asignatura no se considera estrictamente de aplicación didáctica, sino que, desde su descripción inicial, ya está prevista una formación en contenidos geográficos dels propio estudiante, combinada -como siempre en la titulación de Maestro/a- con cierta aplicación didáctica.

Decidimos escoger trabajar los Objetivos más sociales, los que pensamos que más corresponden a una formación geográfica humana y más coherentes con nuestra área de conocimiento. En principio, nuestra selección es: ODS 1, Fin de la pobreza; ODS 2, Hambre cero; ODS 4, Educación de calidad; ODS 5, Igualdad de género; ODS 8, Trabajo digno y Crecimiento económico; ODS 9, Industria e innovación; ODS 10, Reducción de las desigualdades; ODS 11, Ciudades y comunidades sostenibles; ODS 12, Consumo y producción responsables; ODS 16, Paz y justicia.

Los Objetivos que no planteamos como propios de la asignatura (ODS 3, acerca de la salud; ODS 6 sobre el agua y el saneamiento; ODS 7 sobre Energía asequible y no contaminante; ODS 13, Acción por el clima; ODS 14, Vida submarina; ODS 15, Vida de ecosistemas terrestres, ODS 17, Alianzas para lograr los objetivos) pueden surgir en momentos determinados, pero pueden ser dignos de tratamiento más amplios en otras asignaturas del plan de estudios (y creemos que así ocurre).

¿Por qué son importantes? El Objetivo 1 quiere poner fin a la pobreza en todas sus formas $y$ en todo el mundo para 2030. Creemos que el profesorado debe estar informado de las formas de pobreza y ser sensibles a ellas en la escala mundial, regional y local. Exactamente lo mismo, con el Objetivo 2 que pretende poner fin al hambre, lograr la seguridad alimentaria y la mejora de la nutrición y promover la agricultura sostenible. El Objetivo 4 forma parte de la propia formación de nuestros graduados e intentamos plantearlo de forma transversal, con una mínima introducción a 
la educación para la sostenibilidad. El Objetivo 5 quiere lograr la igualdad de género y empoderar a todas las mujeres y las niñas; en una profesión de maestra/o, mayoritariamente de mujeres, el discurso e intercambio para lograr esta meta se hace fundamental. El Objetivo 8 busca promover el crecimiento económico sostenido, inclusivo y sostenible, empleo pleno y productivo y el trabajo decente para todos. El Objetivo 9 le complementa, buscando construir infraestructuras resilientes, promover la industrialización y fomentar la innovación. Los dos objetivos son más alejados del trabajo cotidiano de un/a profesor/a, pero creemos que una ciudadanía activa y sostenible pasa por querer participar en estos temas. El Objetivo 10 pretende reducir la desigualdad en los países y entre ellos. Uno de los objetivos más geográficos, sin duda, que no vemos obligadas a unir -por la premura del tiempo- con los objetivos 1 y 2 . El Objetivo 11 quiere lograr que las ciudades sean inclusivas, seguras, resilientes y sostenibles. Este será uno de los objetivos fundamentales de nuestro curso porque pensamos que un/a maestro/a debe conocer la ciudad desde puntos de vista alternativos y quisiéramos su implicación para conseguir unas ciudades más sostenibles. El Objetivo 12 pretende garantizar modalidades de consumo y producción sostenibles. Pensamos que la educación para el consumo ha de ser un eje transversal de trabajo del profesorado y forma parte de la geografía que queremos tratar. El Objetivo 16 quiere promover sociedades pacíficas e inclusivas para el desarrollo sostenible, facilitar el acceso a la justicia para todos y crear instituciones eficaces, responsables e inclusivas a todos los niveles. Creemos que el profesorado debe tener herramientas para tratar la educación para la paz y la justicia, tanto en formas transversales, como en estudios geográficos a diferentes escalas de análisis.

\subsection{El plan de trabajo}

La amplitud y diversidad de posibilidades de trabajo se hace evidente al plantearnos diseñar la asignatura a través de los ODS seleccionados. El número de horas disponible y la realidad organizativa (horarios, tamaño de los grupos, aulas) nos hace ser realistas y pragmáticas para escoger actividades docentes posibles de llevar a cabo con garantías de reforzar contenidos geográficos y abrir nuevas miradas sociales a diferentes escalas de análisis. Desarrollamos las sesiones con actividades en referencia a los diversos Objetivos, que suelen ser prácticas de aula, en un tiempo corto, y prácticas que requieren más horas de trabajo fuera del aula. Citamos, a modo de ejemplo, las temáticas tratadas durante el curso 2019/20, que se vio trastocado por la pandemia mundial:

- Introducción a los ODS y concepto de educación para la ciudadanía y ciudadanía sostenible.

- Búsqueda y comentario de datos estadísticos acerca de la pobreza y la escasez de alimentos en diferentes escalas de análisis.

- Trabajo de campo en la ciudad y establecimiento de criterios de análisis para decidir el grado de sostenibilidad del espacio público.

- Trabajo de campo en la ciudad para analizar las representaciones y roles de género en el espacio urbano. 
- Reflexión y planteamiento de acciones para un consumo responsable, partiendo del análisis de la publicidad y nuestros hábitos de consumo.

- Identificación de las características de empleo para todas las personas con requerimientos de trabajo digno, estudiando el papel de las empresas a través de la Responsabilidad Social Corporativa.

- Observación de evidencias de paz y de conflicto en la ciudad y entorno social (a través del nomenclátor de calles y edificios singulares)

- Asentamiento del concepto de educación para la sostenibilidad como un camino para una educación de más calidad.

Al finalizar el curso, hemos diseñado una herramienta evaluativa para discernir hasta qué punto nuestras actividades son novedosas para los estudiantes y sobre todo si han contribuido a formarles en ciudadanía sostenible.

\section{Asunción de los ODS por parte del estudiantado}

En el protocolo evaluativo diseñado preguntamos a los estudiantes acerca de cómo les va a influenciar, en sus vidas, el haber conocido (o reafirmado) los ODS y haberlos trabajado en la forma en que lo hemos hecho. Hemos preguntado por cada uno de los ODS estudiados durante el curso, mencionados anteriormente. Damos cuatro opciones de respuesta:

a) Cambiaré algo en mi vida a partir de lo que ahora sé. Y les pedimos que escriban, como mínimo, un ejemplo. Con estas respuestas, recogemos, sus iniciativas para el cambio, vemos sus formas de participación ciudadana.

b) Me ha gustado ser consciente del tema, pero no creo que vaya a cambiar en nada. Con esta respuesta detectamos si no han desarrollado, con lo que hemos trabajado, nuevas ideas, y por tanto están tranquilos con las que ya tenían (y que, en algunos casos, podrían ser ya muy cercanas a las «nuevas» de los anteriores)

c) No percibo que este ODS tenga relación con mi vida. Detectamos falta de empatía o cierta pereza en esforzarse en relacionar temas.

d) Opción libre: escribir cualquier otra respuesta. Damos la opción de explicarnos más o de notificarnos cualquier idea que no conecte con las tres respuestas anteriores. A veces, los que han marcado, la opción b), aprovechan aquí para explicarnos porqué la han escogido. Hemos podido cotejar 79 respuestas: 60 mujeres y 19 hombres. Presentamos los resultados numéricos en la Tabla 1. Pasamos a exponer brevemente los resultados que se desprenden de dicha encuesta de opinión. 


\begin{tabular}{|c|c|c|c|c|c|c|c|c|c|c|c|c|}
\hline & \multicolumn{4}{|c|}{ ODS 1 y 2} & \multicolumn{4}{|c|}{ ODS 5} & \multicolumn{4}{|c|}{ ODS 8} \\
\hline & Mujer & $\%(\mathrm{M})$ & Hombre & $\begin{array}{c}\% \\
\text { (H) }\end{array}$ & Mujer & $\begin{array}{l}\% \\
\text { (M) }\end{array}$ & Hombre & $\%(\mathrm{H})$ & Mujer & $\begin{array}{l}\% \\
\text { (M) }\end{array}$ & Hombre & $\begin{array}{c}\% \\
(\mathrm{H})\end{array}$ \\
\hline $\begin{array}{l}\text { Se proponen } \\
\text { cambios }\end{array}$ & 46 & 76,7 & 8 & 42,1 & 44 & 73,3 & 9 & 47,4 & 19 & 31,7 & 10 & 52,6 \\
\hline $\begin{array}{l}\text { No se pro- } \\
\text { pone ningún } \\
\text { cambio }\end{array}$ & 13 & 21,7 & 9 & 47,4 & 12 & 20,0 & 7 & 36,8 & 29 & 48,3 & 3 & 15,8 \\
\hline $\begin{array}{l}\text { Nada que } \\
\text { ver }\end{array}$ & 1 & 1,7 & 0 & 0,0 & 0 & 0,0 & 1 & 5,3 & 4 & 6,7 & 5 & 26,3 \\
\hline \multirow[t]{3}{*}{ Otros } & 0 & 0,0 & 2 & 10,5 & 4 & 6,7 & 2 & 10,5 & 8 & 13,3 & 1 & 5,3 \\
\hline & \multicolumn{4}{|c|}{ ODS 11} & \multicolumn{4}{|c|}{ ODS 12} & \multicolumn{4}{|c|}{ ODS 16} \\
\hline & Mujer & $\%(\mathrm{M})$ & Hombre & $\begin{array}{c}\% \\
(\mathrm{H})\end{array}$ & Mujer & $\begin{array}{l}\% \\
\text { (M) }\end{array}$ & Hombre & $\%(\mathrm{H})$ & Mujer & $\begin{array}{l}\% \\
\text { (M) }\end{array}$ & Hombre & $\begin{array}{c}\% \\
(\mathrm{H})\end{array}$ \\
\hline $\begin{array}{l}\text { Se proponen } \\
\text { cambios }\end{array}$ & 24 & 40,0 & 8 & 42,1 & 45 & 75,0 & 12 & 63,2 & 21 & 35,0 & 3 & 15,8 \\
\hline $\begin{array}{l}\text { No se pro- } \\
\text { pone ningún } \\
\text { cambio }\end{array}$ & 31 & 51,7 & 8 & 42,1 & 10 & 16,7 & 6 & 31,6 & 33 & 55,0 & 11 & 57,9 \\
\hline $\begin{array}{l}\text { Nada que } \\
\text { ver }\end{array}$ & 0 & 0,0 & 1 & 5,3 & 0 & 0,0 & 0 & 0,0 & 0 & 0,0 & 2 & 10,5 \\
\hline Otros & 5 & 8,3 & 2 & 10,5 & 5 & 8,3 & 1 & 5,3 & 6 & 10,0 & 3 & 15,8 \\
\hline
\end{tabular}

Tabla 1. Asunción de los ODS por parte de los y las estudiantes. Fuente: Propia.

Ante el ODS 1 (Erradicar la pobreza) y el ODS 2 (Hambre cero), los y las estudiantes han mostrado mayoritariamente su decisión de cambiar algunos detalles de sus vidas cotidianas. Nos aparecen muchas respuestas relacionadas con participar en las campañas de recogida de alimentos. Algunos reconocen que ya lo hacían, pero que conocer los datos concretos de pobreza y hambre les reafirma en esa acción. También se cita la colaboración con ONGs concretas, en las dos formas habituales: uniéndose al voluntariado y aportando fondos monetarios. De forma minoritaria, aparecen respuestas que indican que no habrá cambios en sus vidas. Aunque no se pedía en el cuestionario, la mayoría de los que dan esta respuesta quieren aclarar que no habrá cambios porque ya vienen actuando hasta ahora en las mismas direcciones mencionadas, o sea, el haber trabajado los ODS 1 y 2 les ha hecho más conscientes de unas acciones que ya llevan a cabo. No detectamos diferencias a destacar entre las respuestas de los hombres (19) y las mujeres (60).

Ante el ODS 5 (Igualdad de género), las estudiantes, de forma mayoritaria (44 sobre 60) dicen que van a hacer pequeños cambios en sus vidas, o más bien, expresan deseos de intervenir 
en su futura vida profesional. Expresan la necesidad de educar en la igualdad de género y en la prevención de la violencia de género. Nos citan como acciones importantes: organizar charlas y acciones educativas transversales en las aulas; cuestionar los cuentos infantiles que llevan explícita o implícitamente estereotipos de género, así como todo tipo de juegos y juguetes; asistir a manifestaciones y protestas feministas; fijarse más y mejor en los posibles tratos discriminatorios de nuestro entorno y decidirse a intervenir; cambiar el uso del lenguaje e ir evolucionando a un uso de la lengua no sexista e inclusivo de todas las sensibilidades; escoger las canciones que se escuchan, desechando las letras machistas; intervenir activamente en la erradicación de los micro-machismos en el seno de las familias; participar en las redes sociales para la promoción de la igualdad de género. Podemos observar, por tanto, la descripción de un buen número de acciones previstas para trabajar para la igualdad de género, fácilmente aplicables en las vidas de nuestras estudiantes.

En el caso de los hombres (19), los resultados son mucho más igualitarios entre los que nos saben describir alguna acción de cambio (9) y los que declaran que no ven posibilidades de cambio (7). El cambio que más citan los primeros es el uso del lenguaje y apartar los chistes machistas. Algunos también expresan que no harán ningún cambio expreso porque consideran que ya han sido educados en la igualdad de género e intentan, desde pequeños, ser consecuentes con ello.

Ante el ODS 8 (trabajo decente y crecimiento económico) observamos que las estudiantes mayoritariamente (29) declaran que no van a cambiar específicamente nada en sus vidas, más 8 respuestas calificadas de «otras». Las que nos proponen cambios (19) nos dicen: que se informarán más antes comprar productos para saber detalles de las empresas productoras y distribuidoras, en el sentido de si siguen procesos de producción favorables con el medio ambiente y con el trato a los trabajadores (recordamos que hemos estudiado la Responsabilidad Social Corporativa); que se fijarán en las noticias relacionadas con las condiciones de trabajo en las empresas para así actuar en consecuencia; que darán más importancia a los reportajes que denuncian explotación infantil, bajos salarios y contaminación en los procesos de producción y que intentarán desechar esas marcas. Estas son las acciones de cambio que nos proponen 19 estudiantes mujeres, pero vemos que son más (29) las que no ven nada en qué cambiar; incluso muchas de ellas manifiestan que no está en sus manos (sino en la de los empresarios o de los gobiernos, por ejemplo).

En el caso de los hombres (19), los resultados ante el ODS 8 son distintos: mayoritariamente (10) piensan en esas mismas acciones de cambio (conceptualmente, son las mismas acciones descritas por las mujeres) y alguno más (8) piensan que nada va con ellos, que no hay cambios a hacer o que les queda lejos.

Ante el ODS 11 (Ciudades y comunidades sostenibles) observamos resultados muy parecidos entre estudiantes mujeres y hombres. La mitad de ellos (8) y más de la mitad de ellas (31) consideran que no van a cambiar nada en sus vidas para lograr unas ciudades más sostenibles. En oposición, aquellos (8) y aquellas (24) que nos proponen una acción para el cambio centran sus respuestas en dos tipos de acciones: reformar la movilidad (apartar el coche, y utilizar más transporte público y bicicleta) y aumentar el nivel de reciclaje de los residuos, intentando disminuirlos, en primer lugar. Otros tipos de ideas son prácticamente inexistentes en sus respuestas. 
Ante el ODS 12 (Consumo responsable), los resultados también nos ofrecen igualdad entre hombres y mujeres. En este tema, la gran mayoría ( 45 mujeres y 12 hombres) manifiestan que sí pueden hacer pequeños - grandes cambios en sus vidas para mejorar el consumo orientándolo a más responsabilidad y, por tanto, a más sostenibilidad. Sus respuestas se dirigen a aspectos como: consumir menos en todo; saber diferenciar los bienes necesarios de los superfluos; consumir productos locales o de proximidad; analizar la publicidad y sus estrategias, así como la influencia en uno mismo; cuestionar las marcas y su trayectoria empresarial; reducir, reciclar y reutilizar más; apostar por productos ecológicos; minimizar o eliminar el desperdicio de los alimentos.

Ante el ODS 16 (Paz y justicia) observamos una tendencia mayoritaria tanto en mujeres (33) como en hombres (11) a considerar que no pueden actuar en sus vidas para mejorar e implementar este Objetivo; piensan que no está en sus manos, sino en la de los gobiernos o instituciones lejanas a ellos y ellas. Algunos, pocos, citan que lo único que pueden hacer es votar a los partidos políticos que les parezca harán leyes más justas. Las ideas de los que sí piensan que pueden actuar (21 mujeres y 3 hombres) van en las direcciones siguientes: es necesario educar en el conflicto y querrán hacerlo en su vida profesional; participar en organizaciones que trabajan contra diferentes tipos de violencias; aumentar el propio grado de empatía hacia los demás; como futuros docentes, saber valorar más y mejor las diversidades lingüísticas y culturales; saber valorar más la calidad de vida democrática que tenemos los que vivimos aquí y ahora; ser ejemplo de tolerancia y respeto ante las diferencias de todo tipo (creencias, culturas, género, estatus social....) especialmente como futuros docentes; reforzar el diálogo en todas las situaciones sociales; colaborar con una ONG concreta que trate de la paz y la justicia.

\section{Conclusiones}

Nuestra propuesta formativa se basa en la presunción de que trabajar los Objetivos del Desarrollo Sostenible va a contribuir a la Educación para la Ciudadanía del futuro profesorado, en su formación inicial. Pensamos que, trabajando temáticas concretas, las de los ODS, además de aprender o consolidar conocimientos disciplinarios, va a dar procedimientos para actuar como sería deseable en un contexto de la educación para la ciudadanía sostenible.

Con nuestra asignatura «Sociedad y Sostenibilidad» querríamos haber avanzado en la formación en ciudadanía sostenible en nuestros jóvenes estudiantes, querríamos que esa formación se convirtiera, en su vida profesional, en un abanico de recursos para incentivar, también, en los niños y las niñas la ciudadanía sostenible.

Ante los resultados relatados, se desprende que los estudiantes (hombres y mujeres) ven formas de participación ciudadana muy claras en algunos temas (erradicar la pobreza y el hambre, trabajar para la igualdad de género, cambiar los hábitos de consumo). Podemos concluir que su nivel de ciudadanía sostenible en relación con estos tres-cuatro objetivos es potente y decidida. En cambio, les cuesta mucho más encontrar acciones de cambio, sobre todo originales para conseguir unas ciudades más sostenibles, apoyar un trabajo decente y un crecimiento económico responsable y colaborar con los objetivos de paz y justicia. Su nivel de ciudadanía sostenible, en estos 
objetivos, es más incipiente. Siempre, habiendo valorado muy bien, las ideas potentes que surgen en algunos y algunas.

Observamos también, una excesiva fuerza de algunas ideas preconcebidas y asumidas anteriormente (en su período de escolaridad y en su ambiente social). Daremos dos ejemplos. Uno, es el de un cierto rechazo o apatía hacia temáticas que no consideran de su incumbencia como futuros maestros y maestras, como es el caso de la temática del ODS 8 (trabajo decente). El otro ejemplo es algo más sorprendente: la cantidad de estudiantes que no ven cómo cambiar nada para construir ciudades sostenibles y los que sí, se refieren, casi exclusivamente al transporte y al reciclaje. Esa conclusión nos afecta a las profesoras porque hemos dedicado mucho tiempo a la práctica en la ciudad, la observación de plazas, y hemos compartido y discutido con el estudiantado criterios de sostenibilidad contrastados para el espacio público: seguridad, sociabilidad, autonomía, identidad, accesibilidad, calidad, multifuncionalidad (Serra-Salvi, Medir y Servitja, 2019). Finalmente, a pesar de haber realizado excelentes trabajos sobre el nivel de sostenibilidad de las plazas de la ciudad, cuando les preguntamos acerca del Objetivo 11, prácticamente nada de las características anteriores aparece. Con estos dos ejemplos, nos percatamos de lo difícil que es ampliar y construir conocimiento en algunas temáticas totalmente «fuertes» fuera del ámbito académico.

Por tanto, creemos que hemos conseguido desarrollar algunos hábitos de ciudadanía sostenible en estudiantes del grado y esperamos tengan algún recuerdo efectivo y voluntad de aplicación en su futura tarea profesional. Para los casos en que vemos aún muy limitadas las iniciativas de ciudadanía activa, no podemos más que esperar que con el tiempo -nuestros estudiantes son muy jóvenes- vayan ampliándose.

\section{Referencias bibliográficas}

Barry, J. (2006). Resistance is fertile: from environmental to sustainability citizenship. En A. Dobson y D. Bell (Eds.), Environmental citizenship (pp. 21-48). Cambridge: MIT Press.

Cercadillo, L. (2016). Educación histórica y competencia global. En R. López-Facal (Ed.), Ciencias sociales, educación y futuro. Investigaciones en didáctica de las ciencias sociales. Libro de Actas el VII Simposio de Didáctica de las Ciencias Sociales en el ámbito Ibeoamericano (pp. 216-227). Santiago de Compostela. Recuperado de: http://www.7simposiodcs.com/

García-Pérez, F.F., de Alba-Fernández, N., y Navarro-Medina, E. (2015). La formación inicial del profesorado para enseñar ciudadanía. Experiencias en los niveles de grado y de máster. En B. Borghi, F.F. García-Pérez y O. Moreno-Fernández (Eds.), Novi Cives. Cittadini dallinfanzia in poi (pp. 137-148). Bologna: Patron.

Granados, J. (2004). La didàctica de les ciències socials i la sostenibilitat. Línies de recerca en educació per a la sostenibilitat i l’ensenyament de la geografía al Regne Unit. En R. Batllori, A.E. Gómez, M. Oller y J. Pagès (Eds.), De la teoria... a laula. Formació del professorat $i$ ensenyament de les ciències socials (pp. 228-237). Bellaterra: Universitat Autònoma de Barcelona. 
Granados, J. (2008). Educación para una ciudadanía sostenible en la formación inicial del profesorado. En R.M. Ávila, A. Cruz, M. C. Díez (Eds.), Didáctica de las Ciencias Sociales, Currículo Escolar y Formación del Profesorado. La didáctica de las Ciencias Sociales en los nuevos planes de estudio (pp. 217-229). Jaén: Universidad de Jaén y Asociación Universitaria del profesorado de Didáctica de las Ciencias Sociales.

Mayer, M. (2002). Ciutadans del barri i del planeta. En F. Imbermón (Coord.), Cinc ciutadanies per a una nova educació (pp. 81-102). Barcelona: Graó

Mesa, M. (2019). La educación para la ciudadanía global y los Objetivos de Desarrollo Sostenible: una agenda para la transformación social. Revista Internacional de Educación para la Justicia Social, 8(1), 7-11

Pagès, J. (2007). La enseñanza de las ciencias sociales y la educación para la ciudadanía en España. Didáctica Geográfica, 9, 205-214

Schenetti, M., y Guerra, E. (2018). Educare nellambiente per costruire cittadinanza attiva. Investigación en la escuela. Revista de investigación e innovación educativa, 95, 15-29 doi: http://dx.doi.org/10.12795/IE.2018.i95.02

Serra-Salvi, A., Medir, R.M., y Servitja, L. (2019). Analizar la sostenibilidad de los espacios públicos: estudio de caso para un aprendizaje de ciudadanía global. En X. C. Macía, F.X. Armas y F. Rodríguez (Coords.), La reconfiguración del medio rural en la sociedad de la información. Nuevos desafíos en la educación geográfica (pp. 251-260). Andavira Editora

Souto, X.M. (1998). Didáctica de la geografía. Problemas sociales y conocimiento del medio. Barcelona: Ediciones del Serbal.

UNESCO (2015). Educación para la ciudadanía mundial. Temas y objetivos de aprendizaje. París: UNESCO. Recuperado de: https://unesdoc.unesco.org/ark:/48223/pf0000233876 